Europhysics Letters

PREPRINT

\title{
Chiral Correction to the Spin Fluctuation Feedback in two-dimensional $p$-wave Superconductors
}

\author{
J. GORYO ${ }^{1}$ and M. Sigrist ${ }^{2,3}$ \\ 1 Institute for Solid State Physics, University of Tokyo, Kashiwanoha 5-1-5, Kashiwa, \\ Chiba, 277-8581 Japan \\ 2 Yukawa Institute for Theoretical Physics, Kyoto University, Kyoto 606-8502, Japan \\ 3 Institute for Theoretical Physics, ETH-Hönggerberg, 8093 Zürich, Switzerland
}

PACS. 74.20.Rp - Pairing symmetries (other than s-wave).

PACS. 74.25.Ha - Magnetic properties.

\begin{abstract}
We consider the stability of the superconducting phase for spin-triplet $p$-wave pairing in a quasi-two-dimensional system. We show that in the absence of spin-orbit coupling there is a chiral contribution to spin fluctuation feedback which is related to spin quantum Hall effect in a chiral superconducting phase. We show that this mechanism supports the stability of a chiral $p$-wave state.
\end{abstract}

Cooper pairing in the spin-triplet (odd-parity) channel has been for a long time the privilege of the superfluid ${ }^{3} \mathrm{He}$ only [1]. In the mid-eighties superconductors have been discovered which were considered good candidates for spin-triplet pairing appeared, the so-called heavy Fermion superconductors, e.g. $\mathrm{UPt}_{3}$ and $\mathrm{UBe}_{13}$ [2] More recently, several new compounds such as $\mathrm{UGe}_{2}$ and $\mathrm{Sr}_{2} \mathrm{RuO}_{4}$ have been added to the list of potential spin-triplet superconductors. In particular, in the case the ruthenate we have to date overwhelming experimental evidence of spin-triplet pairing in a state which resembles the A-phase of ${ }^{3} \mathrm{He}$ from point of view of broken symmetries 3 .

Spin-triplet pairing provides even in case of reduced rotation symmetry (crystal field) still a large number of degrees of freedom, which leads in many cases to long lists of potential superconducting phases 沺. An interesting situation occurs in a two-dimensional system, where the weak-coupling approach leads to several spin-triplet pairing states that possess the same condensation energy in the presence of complete spin-rotation symmetry. Systems of this kind are $\mathrm{Sr}_{2} \mathrm{RuO}_{4}$ which has a quasi-two-dimensional (2D) electron band structure [3, 5] and thin films of ${ }^{3} \mathrm{He}$ (whereby we avoid the discussion of the Berezinsky-Kosterlitz-Thouless transition which would occur in a film, see [6]). In both cases the state realized has the structure of a "chiral $p$-wave" state, with an orbital angular momentum pointing out of the plane, $p_{x} \pm i p_{y}$. This chirality has been discussed in connection with a variety of possible effects, such as the zero-field Hall effect [7, 8], modified vortex core states [9] or the spin quantum Hall effect (SQHE) 10 12]. In this letter we will show that chirality may also be an essential part to stabilization of the chiral $p$-wave state through a spin-induced feedback effect.

(C) EDP Sciences 
We study here a 2D system with complete cylindrical rotation symmetry where the gap function of the $p$-wave superconducting state can be expressed as a $2 \times 2$-matrix $\Delta_{\alpha \beta}(\mathbf{k})=$ $i\left(\sigma_{\mu} \sigma_{2}\right)_{\alpha \beta} d_{\mu j} \hat{k}_{j}\left(d_{\mu j}\right.$ is a complex order parameter $(\mu=1,2,3, j=x, y), \sigma^{\mu}$ are the Pauli spin matrices (summation runs over repeated indices) and $\hat{\mathbf{k}}=\mathbf{k} /|\mathbf{k}|$ ). In the weak-coupling approach the condensation energy of a pairing state depends only on the magnitude and shape of its quasi-particle gap, and states with the same gap are degenerate. It is easy to see that the most stable states in our case are the ones with an isotropic gap, $|\Delta|^{2}=d_{\mu i}^{*} d_{\mu j} \hat{k}_{i} \hat{k}_{j}$. There are several different states with the same isotropic gap which we may classify into two groups,

$$
\begin{aligned}
& \left|d_{\mu x}\right|=\left|d_{\mu y}\right| \text { and } \operatorname{Re}\left(d_{\mu x}^{*} d_{\mu y}\right)=0 \quad \text { A-phase } \\
& \left|d_{\mu x}\right| \neq\left|d_{\mu y}\right| \text { and } \operatorname{Im}\left(d_{\mu x}^{*} d_{\mu y}\right)=0 \quad \text { B-phase }
\end{aligned}
$$

which are analogues of the A- (chiral) and B-phase of ${ }^{3} \mathrm{He}$, respectively [1,6]. Obviously, both types of states possess an isotropic gap. While spin-orbit coupling would lift the weak-coupling degeneracy [14, we ignore here this aspect and concentrate on the role of the feedback effect. The feedback mechanism is based on the concept that the modification of the pairing interaction caused by the appearance of the superconducting condensate strengthens or weakens a particular pairing channel. The spin fluctuation feedback effect has been proposed as the mechanism for the stability of the A-phase in superfluid ${ }^{3}$ He under pressure [1, 15. Recently, we have shown that for charged particles an additional, though rather small, feedback effect exists which based on the orbital chirality stabilizes the A-phase [16]. The problem of the weak-coupling degeneracy of the spin-triplet state is the motivation to revisit also the spin fluctuation based feedback mechanism.

We consider an electron system with a quasi-2D parabolic band $\epsilon(\mathbf{k})=\hbar^{2}\left(k_{x}^{2}+k_{y}^{2}-k_{\mathrm{F}}^{2}\right) / 2 m$, where $k_{\mathrm{F}}$ is the Fermi wave number. We introduce the following spin-dependent two-particle interaction

$$
\begin{aligned}
H_{i n t} & =\frac{1}{2 \Omega} \sum_{\mathbf{k}_{1}, \mathbf{k}_{2}, \mathbf{q}} V_{\mathbf{k}_{1}, \mathbf{k}_{2}, \mathbf{q} ; \alpha \beta \gamma \delta} c_{\mathbf{k}_{1}+\mathbf{q} \alpha}^{\dagger} c_{\mathbf{k}_{2}-\mathbf{q} \beta}^{\dagger} c_{\mathbf{k}_{2} \gamma} c_{\mathbf{k}_{1} \delta} \\
& =\frac{1}{2 \Omega} \sum_{\mathbf{q}}\left[g_{0}(\mathbf{q}) \rho_{\mathbf{q}}^{\mu} \rho_{-\mathbf{q}}^{\mu}+v_{\mathrm{F}}^{-2} g_{1 i j}(\mathbf{q}) J_{i \mathbf{q}}^{\mu} J_{j-\mathbf{q}}^{\mu}\right]
\end{aligned}
$$

where the spin density and spin current operators are defined as

$$
\rho_{\mathbf{q}}^{\mu}=\frac{\hbar}{2} \sum_{\mathbf{k}} c_{\mathbf{k} \alpha}^{\dagger} \sigma_{\alpha \beta}^{\mu} c_{\mathbf{k}+\mathbf{q} \beta},
$$

and

$$
\mathbf{J}_{\mathbf{q}}^{\mu}=\frac{\hbar^{2}}{2} \sum_{\mathbf{k}} \frac{2 \mathbf{k}+\mathbf{q}}{2 m_{e}} c_{\mathbf{k} \alpha}^{\dagger} \sigma_{\alpha \beta}^{\mu} c_{\mathbf{k}+\mathbf{q} \beta},
$$

respectively ( $\Omega$ denotes the volume; we sum over repeated indices.). The first term in Eq.(3) describes the paramagnon exchange and $g_{0}(\mathbf{q})$ is given by

$$
g_{0}(\mathbf{q})=-\frac{I(\mathbf{q})}{1-I(\mathbf{q}) \chi(\mathbf{q})}
$$

where $I(\mathbf{q})$ is the spin exchange interaction $\left(I(\mathbf{q})=I_{0}\left\{1+c\left(q / 2 k_{F}\right)^{2}\right\}\right.$ with $I_{0}$ correspond to the spin-spin contact interaction). The spin susceptibility $\chi(\mathbf{q})$ is defined as $\left\langle T_{\tau} \rho_{\mathbf{q}}^{\mu} \rho_{-\mathbf{q}}^{\nu}\right\rangle_{1 \mathrm{PI}}=$ 
$\chi(\mathbf{q}) \delta^{\mu \nu}$ where $\left\langle T_{\tau} \rho_{\mathbf{q}}^{\mu} \rho_{-\mathbf{q}}^{\nu}\right\rangle_{1 \mathrm{PI}}$ denotes the one-particle irreducible (1PI) diagrams for the static spin-spin correlation in the normal state, and $\left\langle T_{\tau} \cdots\right\rangle$ is the thermal expectation value of operators with the imaginary time-ordered product. The single-loop diagram in our twodimensional model leads to a constant susceptibility $\chi(\mathbf{q})=\chi_{0}$ for $q<2 k_{F}$ so that the $q$-dependence of $g_{0}(\mathbf{q})$ occurs through $I(\mathbf{q})$ for small $q$.

The second term in Eq. (3) results from the spin current-spin current contact interaction, $-\left(I_{1} / 2 v_{F}^{2}\right) \mathbf{J}^{\mu}(\mathbf{x}) \cdot \mathbf{J}^{\mu}(\mathbf{x})$. Including the polarization analogous to the above case, we obtain $g_{1 i j}(\mathbf{q})=g_{1}(\mathbf{q})\left(\delta_{i j}-q_{i} q_{j} / q^{2}\right)$, where

$$
g_{1}(\mathbf{q})=-\frac{I_{1}}{1-\left(I_{1} / v_{\mathrm{F}}^{2}\right) \chi_{c}(\mathbf{q})} .
$$

$\chi_{c}(\mathbf{q})$ is defined via the relation with the 1PI static spin current-spin current correlation as $\left\langle T_{\tau} J_{i \mathbf{q}}^{\mu} J_{j-\mathbf{q}}^{\nu}\right\rangle_{1 \mathrm{PI}}=\chi_{c}(\mathbf{q}) \delta^{\mu \nu}\left(\delta_{i j}-q_{i} q_{j} / q^{2}\right)$. For small $q$ we find [17],

$$
\chi_{c}(\mathbf{q}) \simeq v_{\mathrm{F}}^{2}\left(1+\frac{q^{2}}{4 k_{\mathrm{F}}^{2}}\right) \chi(\mathbf{q}) .
$$

The structure of the interaction and the coupling constants are derived from a short-ranged repulsive two-particle interaction (e.g. Coulomb for charged particles),

$$
\mathcal{H}_{c}=\frac{1}{2 \Omega} \sum_{\mathbf{k}, \mathbf{k}^{\prime}, \mathbf{q}} \sum_{\alpha, \beta} U(\mathbf{q}) c_{\mathbf{k}+\mathbf{q}, \alpha}^{\dagger} c_{\mathbf{k}, \alpha} c_{\mathbf{k}^{\prime}-\mathbf{q}, \beta}^{\dagger} c_{\mathbf{k}^{\prime}, \beta} .
$$

Using the $\mathrm{SU}(2)$-identity $3 \delta_{\alpha \delta} \delta_{\beta \gamma}=2 \sigma_{\alpha \beta}^{\mu} \sigma_{\gamma \delta}^{\mu}+\sigma_{\alpha \delta}^{\mu} \sigma_{\gamma \beta}^{\mu}$ and neglecting the irrelevant terms (which show interactions for the spin singlet pairing channel) we obtain a spin-spin exchange interaction as well as spin current-spin current interaction with the above coupling constants,

$$
\begin{aligned}
& I_{0}=\left.\frac{4}{3 \hbar^{2}} U(\mathbf{q})\right|_{\mathbf{q}=0}, \quad c=\frac{2 I_{1}}{I_{0}} \sim \frac{\left(2 k_{\mathrm{F}} l\right)^{2}}{3}, \\
& I_{1}=-\left.\frac{1}{3} \frac{2}{3 \hbar^{2}}\left(2 k_{F}\right)^{2} \frac{\partial^{2} U(\mathbf{q})}{\partial q_{i}^{2}}\right|_{\mathbf{q}=0} \sim \frac{2}{3}\left(k_{\mathrm{F}} l\right)^{2} I_{0}
\end{aligned}
$$

where $l$ is range of the interaction $V_{c}$ (Thomas-Fermi screening length for Coulomb interaction).

Using Eq.(3) and the BCS decoupling scheme we derive the following self-consistent equations in the weak-coupling limit for the $p$-wave pairing channel, which we assume to be dominant

$$
d_{\mu}(\mathbf{k})=-\frac{1}{\Omega} \sum_{\mathbf{k}^{\prime}} \frac{\hbar^{2}}{4}\left[\tilde{g}_{0}-\tilde{g}_{1}\right] \hat{\mathbf{k}} \cdot \hat{\mathbf{k}}^{\prime} \frac{d_{\mu}\left(\mathbf{k}^{\prime}\right)}{2 E_{\mathbf{k}}^{\prime}} \tanh \frac{E_{\mathbf{k}}^{\prime}}{2 k_{B} T},
$$

where the wave vectors $\mathbf{k}$ and $\mathbf{k}^{\prime}$ are restricted to certain range close to the Fermi surface $\left(|\mathbf{k}|=\left|\mathbf{k}^{\prime}\right|=k_{F}\right)$, defined by a cutoff energy $\epsilon_{c}>\left|\epsilon_{\mathbf{k}}\right|,\left|\epsilon_{\mathbf{k}}^{\prime}\right|\left(d_{\mu}(\mathbf{k})=d_{\mu j} \hat{k}_{j}\right)$. The quasiparticle energy is $E_{\mathbf{k}}=\sqrt{\epsilon_{\mathbf{k}}^{2}+(1 / 2) \operatorname{tr}\left[\Delta_{\mathbf{k}}^{\dagger} \Delta_{\mathbf{k}}\right]}$. The effective coupling constants for the $p$-wave channel are obtained from Eq.(3) through

$$
\tilde{g}_{0}=\left\langle g_{0}\left(\mathbf{k}-\mathbf{k}^{\prime}\right) \hat{\mathbf{k}} \cdot \hat{\mathbf{k}}^{\prime}\right\rangle \quad \text { and } \quad \tilde{g}_{1}=(1 / 2)\left\langle g_{1}\left(\mathbf{k}-\mathbf{k}^{\prime}\right)\right\rangle
$$

where the average is taken over the Fermi surface for both wave vectors $\left(|\mathbf{k}|=\left|\mathbf{k}^{\prime}\right|=k_{F}\right)$. The constant $\left[\tilde{g}_{0}-\tilde{g}_{1}\right]$ is negative so that we find a solution for $p$-wave superconductivity in 
Eq.(8). It is easy to see that both types of pairing states (A- and B-phase) satisfy the identical self-consistent equation.

Let us now consider the correction to the coupling functions $g_{0}(\mathbf{q})$ and $g_{1}(\mathbf{q})$ below $\mathrm{T}_{c}$,

$$
\begin{aligned}
g_{0}(\mathbf{q}) \delta^{\mu \nu} & \rightarrow g_{0}(\mathbf{q}) \delta^{\mu \nu}+\delta g_{0}^{\mu \nu}(\mathbf{q}), \\
g_{1 i j}(\mathbf{q}) \delta^{\mu \nu} & \rightarrow g_{1 i j}(\mathbf{q}) \delta^{\mu \nu}+\delta g_{1 i j}^{\mu \nu}(\mathbf{q}) .
\end{aligned}
$$

It is sufficient to restrict ourselves to temperatures close to $T_{c}$ so that we can restrict to the lowest order contributions of the order parameters [18]. Thus, we find,

$$
\begin{aligned}
\delta g_{0}^{\mu \nu}(\mathbf{q})= & \left\{g_{0}(\mathbf{q})\right\}^{2} \delta \pi_{00}^{\mu \nu}(\mathbf{q}) \\
= & C\left\{g_{0}(\mathbf{q})\right\}^{2} f(\mathbf{q})\left\{\delta_{\mu \nu} d_{\rho l}^{*} d_{\rho l}-2 \operatorname{Re} d_{\mu l}^{*} d_{\nu l}\right\} \\
\delta g_{1 i j}^{\mu \nu}(\mathbf{q})= & \left\{g_{1}(\mathbf{q})\right\}^{2} \delta \pi_{i j}^{\mu \nu}(\mathbf{q}) / v_{\mathrm{F}}^{2} \\
= & C\left\{g_{1}(\mathbf{q})\right\}^{2}\left(\delta_{i j}-\frac{q_{i} q_{j}}{q^{2}}\right) h(\mathbf{q}) \\
& \times\left\{\delta_{\mu \nu} d_{\rho l}^{*} d_{\rho l}-2 \operatorname{Re} d_{\mu l}^{*} d_{\nu l}\right\} .
\end{aligned}
$$

where $\delta \pi^{\mu \nu}(\mathbf{q})$ and $\delta \pi_{i j}^{\mu \nu}(\mathbf{q})$ are the correction to the spin-spin correlation function and spin current-spin current correlation function, respectively. Here $C=\chi_{0} /\left(4 \pi k_{B}^{2} T_{c}^{2}\right)$, and

$$
\begin{aligned}
& f(\mathbf{q})=1 / \sqrt{1+\xi^{2} q^{2}}, \\
& h(\mathbf{q})=\left(1+q^{2} / 4 k_{\mathrm{F}}^{2}\right) f(\mathbf{q}) .
\end{aligned}
$$

with $\xi=v_{\mathrm{F}} / 2 \pi k_{\mathrm{B}} T_{\mathrm{c}}$. Note that the approximative analytic form of the function $f(\mathbf{q})$ can be obtained in an analogous way as discussed in Ref. 116]. The corrections are calculated by using the anomalous Green function linearized in the gap function: $\mathcal{F}_{\alpha \beta}\left(\mathbf{k}, i \omega_{m}\right)=i \Delta(\mathbf{k})_{\alpha \beta} /\left(\omega_{m}^{2}+\right.$ $\left.\epsilon(\mathbf{k})^{2}\right)$. The modification of the coupling constant in Eq. (8) is

$$
\delta\left(\tilde{g}_{0}^{\mu \nu}+\tilde{g}_{1}^{\mu \nu}\right)=C \gamma^{\mathrm{sf}}\left\{\delta_{\mu \nu} d_{\rho l}^{*} d_{\rho l}-2 \operatorname{Re} d_{\mu l}^{*} d_{\nu l}\right\}
$$

with

$$
\begin{aligned}
\gamma^{\mathrm{sf}}= & \left\langle f\left(\mathbf{k}-\mathbf{k}^{\prime}\right)\left\{g_{0}\left(\mathbf{k}-\mathbf{k}^{\prime}\right)\right\}^{2} \hat{\mathbf{k}} \cdot \hat{\mathbf{k}}^{\prime}\right. \\
& \left.+\frac{1}{2} h\left(\mathbf{k}-\mathbf{k}^{\prime}\right)\left\{g_{1}\left(\mathbf{k}-\mathbf{k}^{\prime}\right)\right\}^{2}\right\rangle_{|\mathbf{k}|=\left|\mathbf{k}^{\prime}\right|=k_{F}} \\
\sim & \frac{\sqrt{2}}{k_{\mathrm{F}} \xi} \ln \left(k_{\mathrm{F}} \xi\right)\left[\left(g_{0}(0)\right)^{2}+\frac{1}{2}\left(g_{1}(0)\right)^{2}\right],
\end{aligned}
$$

where we have used that the function $f(q)$ is small for $q \gg \xi^{-1} \gg k_{\mathrm{F}}$. The effect depends on the order parameter structure via $\left\{\delta_{\mu \nu} d_{\rho l}^{*} d_{\rho l}-2 \operatorname{Re} d_{\mu l}^{*} d_{\nu l}\right\}$ which is selective for different pairing states.

Now we turn to a different process contributing to the feedback effect which is based on the anomalous coupling of spin density-spin current leading an interaction of the form $H_{\text {an }}=\Omega^{-1} \sum_{\mathbf{q}} \delta g_{0 i}^{\mu \nu}(\mathbf{q}) \rho_{\mathbf{q}}^{\mu} J_{i-\mathbf{q}}^{\nu} / v_{\mathrm{F}}$. Such a coupling cannot exist in the normal state but only occurs as a result of broken time reversal symmetry and parity, as is the case for the chiral A-phase in Eq.(2). The coupling function $\delta g_{0 i}^{\mu \nu}(\mathbf{q})$ to lowest order of the order parameter has the form

$$
\begin{aligned}
\delta g_{0 i}^{\mu \nu}(\mathbf{q})= & \left\{g_{0}(\mathbf{q}) g_{1}(\mathbf{q})\right\} \delta \pi_{0 i}^{\mu \nu}(\mathbf{q}) / v_{\mathrm{F}} \\
= & C\left\{g_{0}(\mathbf{q}) g_{1}(\mathbf{q})\right\} i \epsilon_{i j} q_{i} f(\mathbf{q}) / k_{\mathrm{F}} \\
& \times \epsilon_{k l}\left\{\delta_{\mu \nu} \operatorname{Im} d_{\rho k}^{*} d_{\rho l}-2 \operatorname{Im} d_{\mu k}^{*} d_{\nu l}\right\}
\end{aligned}
$$


where $\delta \pi_{0 i}^{\mu \nu}(\mathbf{q})=\left\langle T_{\tau} \rho_{\mathbf{q}}^{\mu} J_{i-\mathbf{q}}^{\nu}\right\rangle$ shows the spin-spin current correlation function in the superconducting state, calculated by using the linearized anomalous Green function. Analogous to Ref. [16], we find that the effective interaction for $p$-wave pairing channel has the form,

$$
\begin{aligned}
V_{\mathbf{k}, \mathbf{k}^{\prime}, \alpha \beta \gamma \delta}^{\mathrm{an}}= & \frac{\hbar^{2} C}{4}\left(\sigma^{\mu}\right)_{\alpha \delta}\left(\sigma^{\nu}\right)_{\beta \gamma} \gamma^{\text {an }}\left\{i \hat{\mathbf{k}} \times \hat{\mathbf{k}}^{\prime}\right\} \\
& \times \epsilon_{k l}\left\{\delta_{\mu \nu} \operatorname{Im} d_{\rho k}^{*} d_{\rho l}-2 \operatorname{Im} d_{\mu k}^{*} d_{\nu l}\right\},
\end{aligned}
$$

where

$$
\begin{aligned}
\gamma^{\text {an }} & =\left\langle g_{0}\left(\mathbf{k}-\mathbf{k}^{\prime}\right) g_{1}\left(\mathbf{k}-\mathbf{k}^{\prime}\right) f\left(\mathbf{k}-\mathbf{k}^{\prime}\right)\right\rangle_{|\mathbf{k}|=|\mathbf{k}|^{\prime}=k_{\mathrm{F}}} \\
& \sim \frac{\sqrt{2}}{k_{\mathrm{F}} \xi} \ln \left(k_{\mathrm{F}} \xi\right) g_{0}(0) g_{1}(0) .
\end{aligned}
$$

Note that the dependence on the order parameter is different from that in Eq. (13) and that indeed only the chiral pairing state generates this contribution.

The total feedback contribution to be added to the $p$-wave gap equation (8) is given by

$$
\begin{aligned}
\delta d_{\mu i}= & -\frac{1}{\Omega} \sum_{\mathbf{k}^{\prime}} \frac{\hbar^{2} C}{4}\left[\gamma^{\text {sf }} \delta_{i j}\left\{\delta_{\mu \nu} d_{\rho l}^{*} d_{\rho l}-2 \operatorname{Re} d_{\mu l}^{*} d_{\nu l}\right\}\right. \\
& \left.-i \gamma^{\mathrm{an}} \epsilon_{i j}\left\{\delta_{\mu \nu} \operatorname{Im}\left(\epsilon_{k l} d_{\rho k}^{*} d_{\rho l}\right)-2 \operatorname{Im}\left(\epsilon_{k l} d_{\mu k}^{*} d_{\nu l}\right)\right\}\right] \\
& \times \frac{d_{\nu j}}{2 E_{\mathbf{k}^{\prime}}} \tanh \frac{E_{\mathbf{k}^{\prime}}}{2 k_{B} T},
\end{aligned}
$$

Note that both $\gamma^{\text {sf }}$ and $\gamma^{\text {an }}$ are positive and are strong coupling corrections as indicated by the factor $\left(k_{F} \xi\right)^{-1}(\ll 1)$.

The relative magnitude of the two feedback contributions depends on the parameter $k_{F} l$, i.e. on the range of the interaction $U$.

$$
\frac{\gamma^{a n}}{\gamma^{s f}} \sim \frac{g_{0}(0) g_{1}(0)}{g_{0}(0)^{2}+g_{1}(0)^{2} / 2} \sim\left(2 k_{\mathrm{F}} l\right)^{2}\left(1-I_{0} \chi_{0}\right)
$$

where the last analytic form is valid for $k_{\mathrm{F}} l$ much smaller than 1 . Since, however, the range of the interaction can be comparable with the average interparticle distance, this ratio could of order 1 as well and the anomalous contribution can be comparable to the ordinary spin fluctuation feedback. In Fig. 1, we plot $I_{0} \chi_{0}$ dependence of the ratio $\gamma^{a n} / \gamma^{s f}$ in the case $k_{\mathrm{F}} l \sim 1$.

The feedback effect enters as a correction to the fourth-order terms in the Ginzburg-Landau free energy. These terms are readily obtained from Eq.(18) as

$$
\begin{aligned}
\Delta F^{\mathrm{fb}}= & \Gamma^{\mathrm{sf}} \operatorname{Re}\left(d_{\mu i}^{*} d_{\nu i}\right)\left\{\delta_{\mu \nu} d_{\rho l}^{*} d_{\rho l}-2 \operatorname{Re}\left(d_{\mu l}^{*} d_{\nu l}\right)\right\} \\
& +\Gamma^{\mathrm{an}} \operatorname{Im}\left(\epsilon_{i j} d_{\mu i}^{*} d_{\nu j}\right)\left\{\delta_{\mu \nu} \operatorname{Im}\left(\epsilon_{k l} d_{\rho k}^{*} d_{\rho l}\right)\right. \\
& \left.-2 \operatorname{Im}\left(\epsilon_{k l} d_{\mu k}^{*} d_{\nu l}\right)\right\} .
\end{aligned}
$$

with

$$
\begin{aligned}
\Gamma^{\mathrm{sf}} & =\frac{C}{4 \hbar^{2}} \frac{\gamma^{s f}}{\left(\tilde{g}_{0}-\tilde{g}_{1}\right)^{2}} \\
\Gamma^{\mathrm{an}} & =\frac{C}{4 \hbar^{2}} \frac{\gamma^{a n}}{\left(\tilde{g}_{0}-\tilde{g}_{1}\right)^{2}}
\end{aligned}
$$


EUROPHYSICS LETTERS

As we mentioned previously, the part of $\Gamma^{\text {sf }}$ is equivalent to the standard spin fluctuation feedback as discussed in literature [1, 6, 15]. As is well known it modifies the condensation energy to stabilize the (chiral) A-phase in Eq.(2) analogous to superfluid ${ }^{3} \mathrm{He}$. The anomalous contribution due to spin density-spin current coupling in the $\Gamma^{\text {an }}$-term yields an additional bias towards the same A-phase. Considering the order parameter dependence in Eq.(20), we see that the feedback benefit only occurs in the chiral condensate. This chiral correction is intimately related to SQHE in the chiral superconductors or superfluid [10, 11]. SQHE is an effect where a the spin current is induced transverse to the gradient of a Zeeman field, i.e. $\mathbf{J}^{\mu} \cdot \nabla B^{\mu}=0$ where $\mu$ denotes the spin component. This effect has been discussed for states like the A-phase. It was shown that a Chern-Simons term exists in the effective action for the $\mathrm{SU}(2)$ gauge field $\left(A_{0}^{(\mu)}, \mathbf{A}^{(\mu)}\right)$ [12] (the time component $A_{0}^{(\mu)}$ corresponds to the Zeeman field). The SU(2) Chern-Simons term has a bilinear part of the gauge field with one space-time derivative and totally anti-symmetric with respect to space-time indices, i.e. $\kappa \epsilon_{\alpha \beta \gamma} A_{\alpha}^{(\sigma)} \partial_{\beta} A_{\gamma}^{(\sigma)}$ [19], where the subscripts $(\alpha, \beta, \gamma)$ denote space-time indices. The coupling constant $\kappa$ corresponds to the transverse conductance for the spin degree of freedom and is obtained form the spin-spin current correlation $\pi_{0 j}^{\mu \nu}(\mathbf{q})$, which plays an essential role to introduce the chiral correction (Eq.(15)), by calculating

$$
\kappa=\left.\frac{1}{2 ! 2 !} i \epsilon_{i j} \delta^{\mu \nu} \frac{\partial}{\partial q_{i}} \pi_{0 j}^{\mu \nu}(\mathbf{q})\right|_{\mathbf{q}=0} .
$$

It was shown that at zero-temperature $\kappa$ is universal $1 / 2 \pi$ in the units $\mu_{B}$, the Bohr magneton, in the chiral $p$-wave states in contrast to the related charge Hall effect which is non-universal [8, 11, 12].

In summary, we have analyzed the anomalous contribution to the spin feedback effect in spin triplet superconductors or superfluid in a two-dimensional system. This effect together with the standard spin fluctuation feedback effect favors the chiral $p$-wave phase, corresponding to the A-phase in Eq.(2), and lifts in this way the weak-coupling degeneracy among several spin-triplet pairing states. Chirality plays a similar role in the charge chiral feedback effect which is, however, much weaker than the spin chiral feedback effect, because in the former the physical U(1) gauge field mediates the modified interaction [16]. The degeneracy between the A- and B-phase like pairing states is also lifted by spin-orbit coupling [1, 6, 14]. Since this yields a correction to the second order term in the free energy it is generally more decisive. Nevertheless, our discussion shows that in 2D systems where the feedback effect is the decisive mechanism to lift the degeneracy the anomalous coupling between spin-density and spincurrent can give an sizeable contribution.

The authors are grateful to A. Furusaki, M. Kohmoto, H. Kohno, T. Morinari, N. Nagaosa and M. Sato for helpful discussions. This work has been financially supported by a Grant-inAid of the Japanese Ministry of Education, Science, Culture and Sports.

\section{REFERENCES}

[1] A.J. Leggett, Rev. Mod. Phys. 47, 331 (1975). D. Vollhardt and P. Wölfle, The Superfluid Phases of Helium 3, Taylor \& Francis, London (1990).

[2] R.H. Heffner and M.R. Norman, Comments Cond. Mat. Phys. 17, 361 (1996). 
[3] Y. Maeno, et. al. Nature 372 532-534 (1994); G. M. Luke, et. al. ibid. 394, 558-561 (1998); K. Ishida, et. al. ibid. 396, 658 (1998).

[4] L.P. Gor'kov, Sov. Sci. Rev. A Phys. 9, 1 (1987); M. Sigrist and K. Ueda, Rev. Mod. Phys. 63, 239 (1991).

[5] T. M. Rice and M. Sigrist, J. Phys.: Condens. Matter 7, L643 (1995).

[6] S.E. Korshunov, Zh. Eksp. Teor. Fiz. 89, 531 (1985)[Sov. Phys. JETP 62, 301 (1985)].

[7] G.E. Volovik, Sov. Phys. JETP 67, 1804 (1988).

[8] J. Goryo and K. Ishikawa, Phys. Lett. A 260, 294 (1999); J. Goryo and K. Ishikawa, J. Phys. Soc. Jpn. 67, 3006 (1998); A. Furusaki, et. al, cond-mat/0102143.

[9] M. Matsumoto and M. Sigrist, J. Phys. Soc. Jpn. 68724 (1999); G. E. Volovik, JETP Lett. 70 792 (1999); J. Goryo, Phys. Rev. B 614222 (2000); J. Phys. Soc. Jpn, 693501 (2000); Y.Kato, ibid. 693378 (2000).

[10] G.E. Volovik and V.M. Yakovenko, J. Phys.: Condens. Matter 1, 5263 (1989).

[11] T. Sentil, J.B. Marston and M.P.A. Fisher, Phys. Rev. B 60, 4245 (1999).

[12] N. Read and D. Green, Phys. Rev. B 61 (2000).

[13] M. Sigrist et. al., Physica C 317-318 134 (1999).

[14] K. K. Ng and M. Sigrist, Europhys. Lett. 49, 473 (2000).

[15] W. F. Brinkman, et. al, Phys. Rev. A 102386 (1974). Y. Kuroda Prog. Theo. Phys. 53349 (1975).

[16] J. Goryo and M. Sigrist, J. Phys. CM 12 L599 (2000).

[17] Here, we use a relation for small $q$

$$
\begin{aligned}
\left\langle T_{\tau} J_{i \mathbf{q}}^{a} J_{j-\mathbf{q}}^{b}\right\rangle_{1 \mathrm{PI}} \simeq & v_{\mathrm{F}}^{2}\left\{1+\left(\frac{q}{2 k_{\mathrm{F}}}\right)^{2}\right\}\left(\delta_{i j}-\frac{q_{i} q_{j}}{q^{2}}\right) \\
& \times\left\langle T_{\tau} \rho_{\mathbf{q}}^{a} \rho_{-\mathbf{q}}^{b}\right\rangle_{1 \mathrm{PI}},
\end{aligned}
$$

which is verified at the 1-loop level.

[18] We also restrict our discussion to the static part for simplicity. The inclusion of dynamical contributions does not change the result qualitatively, but makes the analysis considerably more complicated.

[19] S. Deser, R. Jackiw, and S. Templeton, Ann. Phys. (NY) 140, 372 (1982) ; Phys. Rev. Lett.48, 975 (1982) 
EUROPHYSICS LETTERS

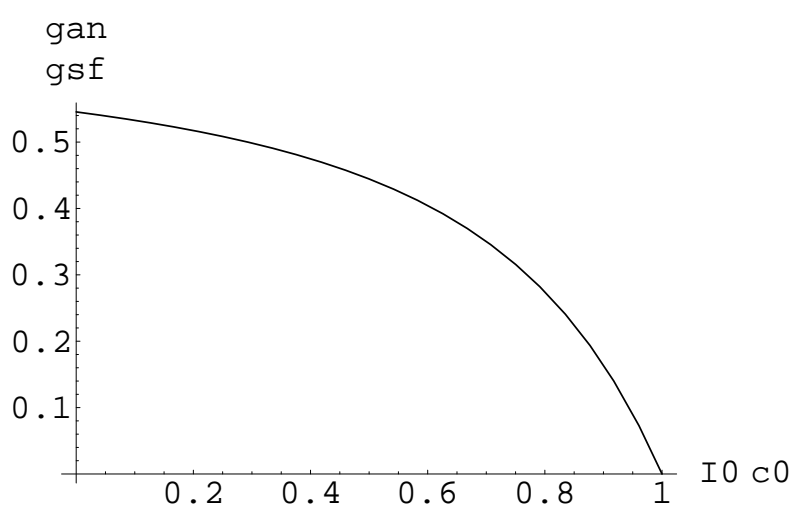

Fig. $1-I_{0} \chi_{0}$ dependence of the ratio $\gamma_{a n} / \gamma_{s f}$ (Eq. (19) ) in the case $k_{\mathrm{F}} l \sim 1$. 